Discrete Comput Geom 30:299-309 (2003)

DOI: $10.1007 / \mathrm{s} 00454-003-0011-\mathrm{x}$

\title{
The Complexity of Hyperplane Depth in the Plane
}

\author{
Stefan Langerman ${ }^{1}$ and William Steiger ${ }^{2}$ \\ ${ }^{1}$ Computer Science, McGill University, \\ Montreal, Quebec, Canada H3A 2A7 \\ sl@cgm.cs.mcgill.ca \\ ${ }^{2}$ Department of Computer Science, Rutgers University, \\ Piscataway, NJ 08854-8019, USA \\ steiger@cs.rutgers.edu
}

\begin{abstract}
Given a set of $n$ hyperplanes $h_{1}, \ldots, h_{n} \in R^{d}$ the hyperplane depth of a point $P \in R^{d}$ is the minimum number of hyperplanes that a ray from $P$ can meet. The hyperplane depth of the arrangement is the maximal depth of points $P$ not in any $h_{i}$. We give an optimal $O(n \log n)$ deterministic algorithm to compute the hyperplane depth of an arrangement in dimension $d=2$.
\end{abstract}

\section{Introduction and Summary}

Given a set $S=\left\{h_{1}, \ldots, h_{n}\right\}$ of $n$ real numbers, the depth of $x \in R$ is defined to be

$$
d(x)=\min \left(\left|\left\{h_{i} \leq x\right\}\right|,\left|\left\{h_{i} \geq x\right\}\right|\right),
$$

and may be thought of as the "size" of the smallest half-line containing $x$. A median is a point of maximal depth $(\lceil(n+1) / 2\rceil)$ and may be found in $O(n)$ time.

There are many situations - for example in Statistics - where multivariate generalizations of ranks and order statistics are useful, and several proposals for depth in $R^{d}$ have been made [2], [7]. In one of the most familiar ones [15], we are given a set $S=\left\{P_{1}, \ldots, P_{n}\right\}$ of $n$ points in $R^{d}$. The depth, or Tukey depth, of $x \in R^{d}$ is defined as the minimal number of points of $S$ in a closed half-space containing $x$. A Tukey median is a point of maximal depth. When $d=1$ this notion agrees with the depth in (1), and with the usual median.

It is a well-known consequence of Helly's theorem (e.g., [6]) that there is a point in $R^{d}$ of Tukey depth at least $\lceil n /(d+1)\rceil$; such a point is called a centerpoint. When $d=2$, Cole et al. described an $O\left(n(\log n)^{5}\right)$ algorithm [5] to construct a centerpoint; 
ideas presented by Cole in [3] could be used to improve the complexity to $O\left(n(\log n)^{3}\right)$. Recently, Jadhav and Mukhopadhyay [8] gave a linear-time algorithm for this task.

For the planar Tukey median, Matoušek [11] has described an algorithm that runs in time $O\left(n(\log n)^{5}\right)$. We have improved this result with an $O\left(n(\log n)^{4}\right)$ algorithm, and gave a lower bound of $\Omega(n \log n)$ for the task (see [10]; a full version of that paper further improves the upper bound by an additional factor of $\log n$ ).

Recently, Rousseeuw and Hubert [12] proposed an interesting new notion of depth for $R^{d}$. Given a set $S=\left\{h_{1}, \ldots, h_{n}\right\}$ of $n$ hyperplanes in $R^{d}$, they defined the hyperplane depth of a point $x \in R^{d}$ to be

$$
\delta(x)=\min _{u:\|u\|=1}(r(x, u)),
$$

where $r(x, u)$ is the number of $h_{i} \in S$ that meet the ray $\{x+t u, t \geq 0\}$ through $x$ in direction $u$. A median is a point of maximal depth. When $d=1$, (2) agrees with the usual definition of depth in (1).

Hyperplane depth was motivated by problems in robust regression. Using a familiar point/hyperplane duality transformation, the $h_{i}$ dualize to $n$ data points in $R^{d}$, while the point $x \in R^{d}$ dualizes to a hyperplane $h$, meant to fit the data. The "regression depth" of $h$ is the minimum number of data points $h$ meets in a rotation-to-vertical; this corresponds exactly to the hyperplane depth of $x$ in the original problem.

Hyperplane depth has attracted much recent interest. On the combinatorial side it was shown by Amenta et al. [1] that like the Tukey median, the hyperplane median must have depth at least $\lceil n /(d+1)\rceil$. In fact, the lower bound is sharp, attained when the $h_{i}$ are duals of $n$ points on the moment curve in $R^{d}$ (see [12]). Other interesting combinatorial questions remain open, for example, whether $n$ given hyperplanes in $R^{d}$ can be partitioned into $k=\lfloor n /(d+1)\rfloor$ sets, $d+1$ hyperplanes in each set, in such a way that the $k$ simplices they determine have nonempty intersection. The affirmative was conjectured by Rousseeuw and Hubert [12]; Steiger and Wenger [14] proved that a fraction $c_{d} n$ of $n$ given hyperplanes partition into mutually intersecting simplices, $c_{d}>0$, a (very small) constant depending on $d$ but independent of $n$; Amenta et al. [1] improved the constant to $c_{d}=1 / d$.

On the computational side, a main question is to determine the complexity of selection-computing points of given depth, or of maximal depth. We use the unit cost RAM model. The hyperplanes in $S$ partition $R^{d}$ into a complex of $O\left(n^{d}\right)$ convex cells, called the arrangement of $S$, and written $A(S)$. It is clear that every point in a cell has the same depth. The depth of the arrangement, $\delta(A(S))$, is defined to be the depth of the deepest cell. The task therefore is to compute $\delta(A(S))$, and a witness point of that depth, or to find a cell of given depth $k \leq \delta(A(S))$.

Rousseeuw and Hubert point out that $\delta(x)$ can be computed in time $O\left(n^{d-1} \log n\right)$ for any $x$, and since there are $O\left(n^{d}\right)$ vertices in $A(S)$, selection has cost $O\left(n^{2 d-1} \log n\right)$. For $d=2$ they mention an $O\left(n^{3}\right)$ algorithm [13]. In [1] it was observed that $A(S)$ can be constructed in $O\left(n^{d}\right)$ and then, using breadth-first-search on the graph of adjacent cells, the depth of every cell is obtained in the same $O\left(n^{d}\right)$ time. This is the analogue of using sorting to find the ordinary median.

For the case $d=2$, van Kreveld et al. [9] recently described a beautiful $O\left(n(\log n)^{2}\right)$ algorithm for hyperplane depth in $R^{2}$. This shows that it is not necessary to find the depth 
of every cell in order to find the deepest one and is analogous to the fact that sorting is not optimal for computing the usual median. The algorithm is controlled by a binary search based on being able to select the $k$ th vertex —ordered by $x$-coordinate-in a set of consecutive, unsearched candidate vertices. This may be done in $O(n \log n)$ (see, e.g., [4]). The vertical line through the selected vertex is then analyzed for sidedness. The sidedness test for a line $\ell$ determines one of the two half-planes delimited by $\ell$ that intersects a maximum depth cell, and has complexity $O(n \log n)$. Since there are $O\left(\log \left(\begin{array}{l}n \\ 2\end{array}\right)\right)$ binary search steps, the overall complexity of the algorithm is $O\left(n(\log n)^{2}\right)$. In this paper we prove the following.

Theorem 1. Given a set $S$ of $n$ lines in the plane, $\delta(A(S))$, the hyperplane depth of the arrangement, and a witness point $\mu$ with that depth, can be obtained in $O(n \log n)$ time and $O(n)$ space.

The proof is via an algorithm, shown to run in time $O(n \log n)$. An easy consequence is that in the same time bounds, given $k \leq \delta(A(S))$, a point of depth $k$ can be found. It is interesting that our algorithm combines a light form of parametric search and pruneand-search to improve the result in [9], much like the way that the linear-time algorithm for the usual median improves on sorting.

We are searching for a point $\mu$ in a cell $C$ of maximal depth. In fact, we will determine for each line in $S$ its above/below relationship with $\mu$. Each step of the algorithm solves the parametric problem of finding that above/below relationship for a constant fraction $a<1$ of the $m$ (initially $n$ ) remaining lines. These lines are then pruned out and the search continues with the unpruned lines. We are able to do the pruning step in $O(m \log n+n)$. Since there are at most $m=n(1-a)^{j}$ unpruned candidates after the $j$ th step, and at most $\log n$ steps in all, the complexity is at most

$$
c n \log n+\sum_{j=0}^{\infty} b(1-a)^{j} n \log n=O(n \log n) .
$$

We also prove a matching lower bound for this task in the algebraic decision tree model of computation, thus showing our algorithm to be optimal:

Theorem 2. Given a set $S$ of $n$ lines in $R^{2}$, computing $\delta(A(S))$ requires $\Omega(n \log n)$ steps in any algebraic decision tree.

A similar lower bound can also be stated for the complexity of computing the depth of any given point:

Lemma 1. Given a set of $n$ lines in $R^{2}$, an integer $k$, and a query point $q \in R^{2}$, $\Omega(n \log n)$ steps are needed by an algebraic decision tree that can decide if $\delta(q)>k$.

Finally we point out that although the notion of hyperplane depth is new, already more is known about the complexity of the selection problem using hyperplane depth than is known about selection using Tukey depth. 


\section{Tools}

In this section we describe some tools that are used in the main algorithm. In particular, we describe the sidedness test for a line, and insideness test for a convex closed curve. To simplify the discussion, we define $\tilde{r}(x, u)$ to be the number of lines of $S$ crossed by the ray $\{x+t u, t>0\}$ (the difference with $r$ being that we are not counting lines passing through $x)$. We also define $\tilde{\delta}(x)=\min _{u:\|u\|=1}(\tilde{r}(x, u))$. Note that if $x$ is inside a cell, $\delta(x)=\tilde{\delta}(x)$, and so, since we are looking for a cell of maximum depth, we can use $\tilde{\delta}$ instead of $\delta$ without changing the result.

Given a point $x$, we say that $u$ is a witness direction if $\tilde{r}(x, u)=\tilde{\delta}(x)$. We will need the following:

Lemma 2 (Wedge Lemma [9]). Let $x$ be a point and let $u$ and $v$ be directions of rays from $x$. Then no cell of $A(S)$ intersecting the wedge (cone) formed by $u$ and $v$ at $x$ has depth greater than $\max (\tilde{r}(x, u), \tilde{r}(x, v))$.

Given a point $x$, we call the wedge at $x$ the set of all directions that can be formed by positive linear combinations of witness directions at $x$. By the Wedge Lemma, no cell that intersects the wedge at $x$ can have depth greater than $\tilde{\delta}(x)$. Note also that if the wedge at $x$ contains all directions, then $x$ is of maximum depth. Otherwise, the wedge at $x$ forms an angle less than $\pi$. Here are a few more facts worth noticing:

- If $x$ is on a segment separating two adjacent cells and $x^{+}$and $x^{-}$are points in the adjacent cells, then $\tilde{\delta}(x)=\min \left(\tilde{\delta}\left(x^{+}\right), \tilde{\delta}\left(x^{-}\right)\right)$.

- On any path in the plane, points $x \neq y$ that have no line of $S$ separating them are in the same cell, and have the same wedges. On any path in the plane (not touching any vertex of the arrangement), any two consecutive wedges intersect.

Using the Wedge Lemma, van Kreveld et al. [9] described a sidedness test for a line $\ell$. In time $O(n \log n)$ it computes $k=\max (\delta(x), x \in \ell)$ and gives a side (left or right) of $\ell$ which does not contain a point of depth $>k$.

We now describe a new way to test sidedness based on the following insideness test. Suppose we are given a convex polygon $\mathcal{P}$ whose boundary does not contain a vertex of $A(S)$, and such that no vertex of $\mathcal{P}$ is on a line of the arrangement. Let $k$ denote the depth of the deepest point on the boundary of $\mathcal{P}$. We want to determine which of the inside or the outside of the convex polygon cannot contain a cell of depth greater than $k$. Note that if $k=\delta(A(S))$ is the depth of the arrangement, any answer would be correct.

Lemma 3. Given a convex polygon $\mathcal{P}$ with s sides and a subset $U=\left\{\ell_{1}, \ldots, \ell_{m}\right\}$ of the lines of $S$ that meet the interior of $\mathcal{P}$, then the insideness test for $\mathcal{P}$ can be performed in $O(m \log n+s)$.

Proof. We continuously move a point $x$ around the boundary of $\mathcal{P}$, starting at the leftmost vertex, and keeping track of the wedge at $x$. Note that the wedge only changes when $x$ crosses a line of $U$. In [9] a data structure is presented to maintain the wedge at $x$ as $x$ moves along a path. Its cost is $O(\log n)$ per line crossing. At the same cost it gives 


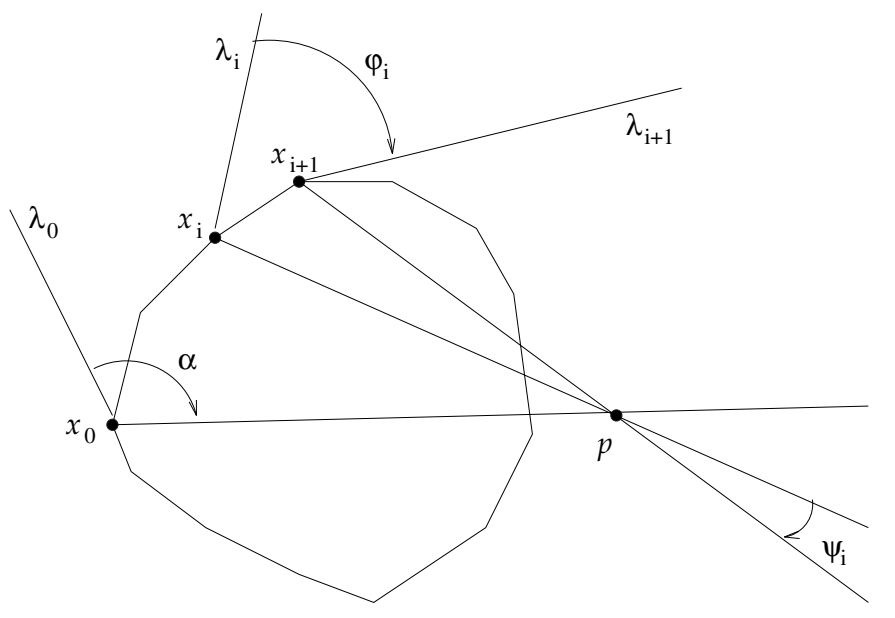

Fig. 1. Insideness test.

$\max (\delta(x))$ for the points traversed, so $k$, the greatest depth of points on the boundary of $\mathcal{P}$, is computed in the traversal of $\mathcal{P}$. The number of wedges is $4 m$ since each line crosses the boundary of the polygon twice, and there are two wedges per line crossing, one when $x$ moves onto a new line, and another when it passes that line. Therefore the cost of the traversal is $O(m \log n)$.

Let $x_{0}, \ldots, x_{4 m-1}, x_{4 m}=x_{0}$ be $4 m$ points on the path that mark the wedge change events (see Fig. 1). Observe the movement of the leftmost ray $\lambda_{i}$ of the wedge at $x_{i}$ along the path, and let $-\pi \leq \varphi_{i} \leq \pi, i=0, \ldots, 4 m-1$, be the angle between $\lambda_{i}$ and $\lambda_{i+1}$. Note that since any two consecutive wedges intersect, we can move the ray to its next position while always staying inside a wedge, and any point swept by the ray cannot have depth greater than $k$.

Claim 1. If the outside of the polygon contains a point of depth greater than $k$, then

$$
\sum_{i=0}^{4 m-1} \varphi_{i}=0 .
$$

Proof. If $p$ is a point outside of the polygon of depth greater than $k$, then that point is not swept by the ray, by the Wedge Lemma. Let $0<\alpha<2 \pi$ be the angle $\lambda_{0} x_{0} p$, and let $-\pi \leq \psi_{i} \leq \pi$ be the angle between the two directions $x_{i} p$ and $x_{i+1} p$. By induction, for any $r$, we have

$$
\alpha-2 \pi+\sum_{i=0}^{r} \psi_{i}<\sum_{i=0}^{r} \varphi_{i}<\alpha+\sum_{i=0}^{r} \psi_{i}
$$

because the last movement of the ray cannot sweep $p$. Since $p$ is outside the polygon, and $x_{4 m}=x_{0}$, we have

$$
\sum_{i=0}^{4 m-1} \psi_{i}=0
$$


and so

$$
-2 \pi \leq \alpha-2 \pi<\sum_{i=0}^{4 m-1} \varphi_{i}<\alpha \leq 2 \pi .
$$

However, $\sum_{i=0}^{4 m-1} \varphi_{i}$ must be a multiple of $2 \pi$ since $\lambda_{4 m}=\lambda_{0}$, so it must be 0 .

Claim 2. If the inside of the polygon contains a point of depth greater than $k$, then

$$
\sum_{i=0}^{4 m-1} \varphi_{i} \neq 0
$$

Proof. Same as for the previous claim, but now, since $p$ is inside the polygon, we have

$$
\sum_{i=0}^{4 m-1} \psi_{i}=2 \pi
$$

and so

$$
0 \leq \alpha-2 \pi+2 \pi<\sum_{i=0}^{4 m-1} \varphi_{i}<\alpha+2 \pi .
$$

Thus the sum cannot be 0 .

To complete the proof of Lemma 3, note that we can precompute the ordering of all $2 \mathrm{~m}$ line crossings, walk around the polygon, and compute $k$ and $\Sigma \varphi_{i}$, all in $O(m \log n+s)$ time. If the sum is 0 , then the inside does not contain a point of depth greater than $k$. Otherwise, the outside does not contain a point of depth greater than $k$, and this proves the lemma.

Corollary 1. Suppose we have a line $\ell$, a convex polygon $\mathcal{P}$ with $s$ sides, and a subset $U=\left\{\ell_{1}, \ldots, \ell_{m}\right\} \subseteq S$ of the lines of $S$ that meet the interior of $\mathcal{P}$. If $\mathcal{P}$ contains a cell $C$ of maximal depth, then the sidedness test for $\ell$ can be performed in $O(m \log n+s)$.

Proof. If $\ell$ does not meet $\mathcal{P}$, the sidedness test is trivial. Otherwise let $\mathcal{P}^{+}$denote the part of $\mathcal{P}$ "above" $\ell$, together with the segment on $\ell$ that meets $\mathcal{P}$. The insideness test for $\mathcal{P}^{+}$resolves the sidedness of $\ell$.

Note that if the polygon is chosen to be big enough to contain all the vertices of the arrangement, this test performs the usual sidedness test from [9].

\section{Pruning}

At every step of the algorithm (see Fig. 2), the lines are partitioned into two sets: the set $Q$ contains all the pruned lines, and $U=\left\{\ell_{1}, \ldots, \ell_{m}\right\}$ contains the unpruned lines. 
$\operatorname{PRUNE}(U, Q, \mathcal{P})$

1. if $|U|<c$ then side every line in $U$, update $\mathcal{P}$, and return $\mu \in \mathcal{P}$; else:

2. compute $\ell$, a centerpoint line for $U$.

3. side $\ell$ to determine which side contains $\mu$.

4. find $q_{i}=\ell \cap \ell_{i}, i=1, \ldots, m$, select vertical lines $x=a_{1}, \ldots, x=a_{9}$ dividing the plane into vertical strips, so that each strip contains at most $m / 9$ of the $q_{i}$.

5. side each vertical line $x=a_{j}$ to find a vertical strip $\left(a_{j}, a_{j+1}\right)$ containing $\mu$.

6. find lines $X \subset U$ that meet the vertical through $\mu$ on the side of $\ell$ opposite $\mu$ : set $U^{\prime} \leftarrow$ $U \backslash X, Q^{\prime} \leftarrow Q \cup X$, and $\mathcal{P}^{\prime} \leftarrow \operatorname{conv}(\mathcal{P} \cap \operatorname{conv}(X))$

7. call PRUNE $\left(U^{\prime}, Q^{\prime}, \mathcal{P}^{\prime}\right)$

Fig. 2. Pruning.

Write $A(U)$ for the arrangement of the $m$ unpruned lines. For every line in $Q$, we know if it is above or below some cell of maximal depth, and we maintain the intersection of these half-spaces as a convex polygon $\mathcal{P}$ which contains a cell of maximal depth. At the beginning of the algorithm, $Q$ is empty, and $\mathcal{P}$ is some polygon large enough to contain all the vertices in the arrangement; such a polygon can be constructed in $O(n \log n)$ time. Consider some point $\mu$ in $\mathcal{P}$ of maximal depth, and let $v$ be the vertical line passing through $\mu$. We want to solve the parametric problem of determining for a constant fraction of the lines in $U$, whether those lines cross $v$ above or below $\mu$, but without knowing $\mu$. For a line $\ell$ we say "side $\ell$ " and mean "determine which side of $\ell$ contains $\mu$."

First, in Step 2, we compute the centerpoint line $\ell$ for $U$. This line is the dual of a (usual) centerpoint for the points dual to the lines in $U$. It has the property that it is below the $m / 3$ level of $A(U)$ and above the $2 m / 3$ level. Jadhav and Mukhopadhyay [8] give a linear-time algorithm to compute $\ell$.

Next, in Step 3, we perform the sidedness test for that line $\ell$, restricted to the polygon $\mathcal{P}$. By Corollary 1 this may be done in time $O(m \log n+n)$.

Assume without loss of generality that $\mu$ is below $\ell$. In Step 4 we compute the points $q_{i}=\ell \cap \ell_{i}, i=1, \ldots, m$, and then, using selection by $x$-coordinate, find points $p_{j}=\left(a_{j}, b_{j}\right) \in \ell, j=1, \ldots, 9$, so that (i) $p_{1}$ is to the right of at most $m / 9$ of the $q_{i}$, (ii) $p_{9}$ is to the left of at most $m / 9$ of the $q_{i}$, and (iii) there are at most $m / 9$ of the $q_{i}$ between each adjacent pair $p_{j} p_{j+1}$ (see Fig. 3). Therefore the vertical lines $x=a_{1}, \ldots, x=a_{9}$ divide the plane into ten vertical strips, each containing at most $m / 9$ of the $q_{i}$. We can do this in $O(m)$ using the fast selection algorithm. Now we perform the sidedness test (restricted to $\mathcal{P}$ ) for each of those vertical lines in $O(m \log (n)+n)$ time. This will either find a point of maximal depth, or determine a strip between adjacent verticals that contains the maximal depth point $\mu$.

Claim 3. In Step 5, in $O(m)$ time, the above/below relation may be determined for at least $2 m / 9$ lines $X \subset U$.

Proof. Let $x=a_{i}$ and $x=a_{i+1}$ be the left and right verticals of the strip determined in Step 4 (take $a_{0}=-\infty$ and $a_{10}=\infty$ ). By construction $\ell$ has at most $m / 9$ intersections in 




Fig. 3. Intersections of unpruned lines with $\ell$ and the strip (containing $\leq m / 9$ of them) that contains $\mu$.

$\left(a_{j}, a_{j+1}\right)$ with lines in $U$. In addition there are at least $m / 3$ lines in $U$ that meet $x=a_{j}$ or $x=a_{j+1}$, or both, above the level $m / 3$. If these lines meet $\ell$ outside $\left(a_{j}, a_{j+1}\right)$ they cross $v$ above $\mu$ and can be pruned. It follows that at least $m / 3-m / 9=2 m / 9$ lines of $U$ can be pruned, since they have been determined to be above $\mu$.

In order to prune a line in $X$ in Step 5, we remove it from $U$ and add it to $Q$. We then update the polygon $\mathcal{P}$ in $O(\log n)$ per line using an incremental convex hull algorithm. The overall cost of Steps $2-5$ is therefore $O(m \log n+n)$. When the number of unpruned lines falls below some predefined constant $c$, we can perform the sidedness test to all remaining lines, then update $\mathcal{P}$ and find a contained point in $O(n \log n)$ time. This is the base case covered in Step 1.

Proof of Theorem 1. In view of Claim 3, after $j$ recursive calls to PRUNE we have $m \leq(7 / 9)^{j} n$. Summing over the at most $O(\log n)$ levels of recursion as in (3) completes the proof.

A simpler algorithm would replace the centerpoint-line construction that used Jadhav and Mukhopadhyay's algorithm by a random choice of a line from $U$. With positive constant probability, the intersection of that line with $v$, the vertical line through $\mu$, will have $n / 3$ intersections above and below it. The resulting randomized algorithm would have the same $O(n \log n)$ running time for its expected cost, but a simpler implementation. 


\section{The Lower Bounds}

Here we prove the lower bound of Lemma 1 and Theorem 2. Let $C$ denote the unit circle in $R^{2}$ and let $T$ denote an algebraic decision tree that can decide whether $\delta(A(S)) \geq\lfloor m / 2\rfloor$ for a set $S$ of $m$ lines in $R^{2}$. We restrict attention to lines that are tangent to $C$ and we will even hold most of the lines fixed. Such inputs can be encoded by the arguments $\left(\varphi_{1}, \ldots, \varphi_{m}\right) \in R^{m}$ of the tangency points, written in polar coordinates. We will show that the subset $Y \subseteq R^{m}$ of restricted inputs where $T$ returns YES has $\Omega(m \log m)$ path connected components.

Consider the point $\theta=\left(\theta_{1}, \ldots, \theta_{m}\right)$ where

$$
\theta_{i}=\left(\frac{i}{4 n+3}\right) 2 \pi, \quad i=1, \ldots, 4 n+3 .
$$

This describes $m=4 n+3$ equally spaced points on $C$, no two diametric. In fact the diameter containing $\theta_{i}$ is a halving line-both its open semicircles contain $2 n+1$ of the other $\theta_{j}$. A diameter containing none of the $\theta_{i}$ is a non-halving diameter and has one open half-circle of $C$ with $2 n+1$ points and the other with $2 n+2$ points. The vector $\theta$ will be used to encode the set of lines $S=\left\{\ell_{1}, \ldots, \ell_{m}\right\}$, where $\ell_{i}$ is tangent to $C$ at the point with argument $\theta_{i}$. The maximum depth cell in $A(S)$ is the regular convex $m$-gon $\mathcal{P}$ in which $C$ is inscribed, and is tangent to $C$ at the $\theta_{i}$. For any $x$ in $\mathcal{P}$ every directional depth will be either $2 n+1$ or $2 n+2$, depending on whether or not the direction is orthogonal to a halving diameter, or if it is orthogonal to a non-halving diameter and points to the larger half-circle. Therefore $\delta(A(S))=2 n+1=\lfloor m / 2\rfloor$.

The set of inputs to $T$ that we consider are in a set $I$, where $z=\left(z_{1}, \ldots, z_{m}\right) \in I$ if $z_{i} \in[0,2 \pi], i=1, \ldots, m$, and $z_{j}=\theta_{j}, j \equiv 1,2,3 \bmod 4$. Let $\pi$ and $\rho$ be two different permutations of the integers $4,8, \ldots, 4 n$, and define the input $\theta_{\pi} \in I$ by

$$
\left(\theta_{1}, \theta_{2}, \theta_{3}, \theta_{\pi_{1}}, \ldots, \theta_{4 n-1}, \theta_{\pi_{n}}, \theta_{4 n+1}, \theta_{4 n+2}, \theta_{4 n+3}\right)
$$

and input $\theta_{\rho} \in I$ by

$$
\left(\theta_{1}, \theta_{2}, \theta_{3}, \theta_{\rho_{1}}, \ldots, \theta_{4 n-1}, \theta_{\rho_{n}}, \theta_{4 n+1}, \theta_{4 n+2}, \theta_{4 n+3}\right) ;
$$

the permutations $\pi$ and $\rho$ point to every fourth entry. Both $\theta_{\pi}$ and $\theta_{\rho}$ are in $Y \cap I$, as they describe the $m$ lines in $S$, but in a different order. We argue that they are in different components.

We hold the $\theta_{j}$ fixed for all $j \equiv 1,2,3 \bmod 4$, and move each $\theta_{\pi_{i}}$ continuously on $C$ to the corresponding $\theta_{\rho_{i}}, i=1, \ldots, n$. This describes a continuous path in $I$ from $\theta_{\pi}$ to $\theta_{\rho}$, and every point $z \in I$ on the path describes $m=4 n+3$ lines tangent to $C$, with $3 n+3$ of the lines fixed. Since $\pi \neq \rho$, some $\theta_{4 j}$ must "cross" (i.e., reverse its radial ordering) a neighbor, $\theta_{4 j-1}$ or $\theta_{4 j+1}$ (see Fig. 4). Write $k=(4 j+2 n \bmod m)+1 ; \theta_{k}$ and $\theta_{k+1}$ are the neighbors of the diameter through $\theta_{4 j}$. If $\theta_{4 j}$ crosses a neighbor, it first will cross the diameter through $\theta_{k}$ or the diameter through $\theta_{k+1}$. When this occurs that diameter will have $2 n$ points on one side and $2 n+2$ on the other, and the $m$ lines that are described by this input $z \in I$ will have $\delta(A(S))=2 n$. Thus we have a point on a path from $\theta_{\pi}$ to $\theta_{\rho}$ that is not in $Y \cap I, \theta_{\pi}$ and $\theta_{\rho}$ must be in different components of 


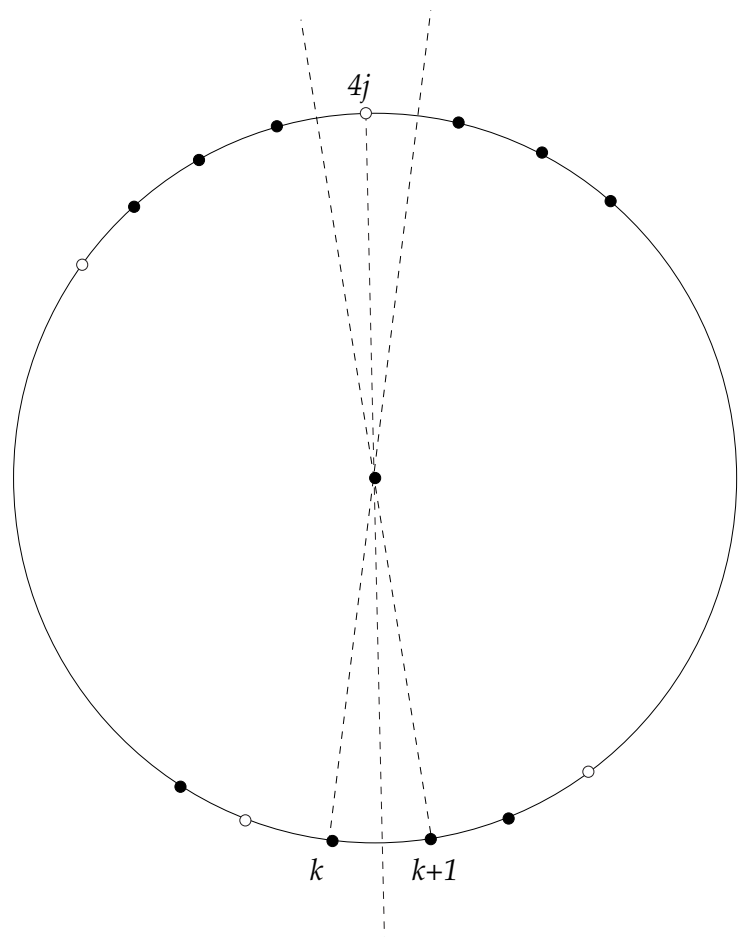

Fig. 4. Lower bound construction.

$Y \cap I$. This accounts for at least $n$ ! different components and since $m=4 n+3$, we get a lower bound of $\Omega(m \log m)$ on the depth of $T$ by Ben Or's theorem [6].

The above argument also establishes an $\Omega(m \log m)$ lower bound for computing the hyperplane depth of a point $x$, so it proves Lemma 1 as well. It would be interesting to know if the same lower bound applies to seemingly easier questions. One is the problem of reporting a point $x$, guaranteed to be in a maximum depth cell of $A(S)$. Another is the problem of reporting the above/below relation of each line with respect to an unknown point $x$ which is in a maximum depth cell.

\section{References}

1. N. Amenta, M. Bern, D. Eppstein, and S.-H. Teng. Regression Depth and Center Points. Discrete Comput. Geom. 23(3) (2000), 305-323.

2. B. Chazelle. New Techniques for Computing Order Statistics in the Euclidean Plane. Proc. First ACM Symposium on Computational Geometry, pp. 125-134, 1985.

3. R. Cole. Slowing Down Sorting Networks to Obtain Faster Sorting Algorithms. J. Assoc. Comput. Mach. 34(1) (1987), 200-208.

4. R. Cole, J. Salowe, W. Steiger, and E. Szemerédi. An Optimal Time Algorithm for Slope Selection, SIAM J. Comput. 18 (1989), 792-810. 
5. R. Cole, M. Sharir, and K. Yap. On k-Hulls and Related Problems. SIAM J. Comput. 16(1) (1987), 61-77.

6. H. Edelsbrunner. Algorithms in Combinatorial Geometry. Springer-Verlag, Berlin, 1987.

7. J. Gill, W. Steiger, and A. Wigderson. Geometric Medians. Discrete Math. 108 (1992), 37-51.

8. S. Jadhav and A. Mukhopadhyay. Computing a Centerpoint of a Finite Planar Set of Points in Linear Time. Discrete Comput. Geom. 12 (1994), 291-312.

9. M. van Kreveld, J. Mitchell, P. Rousseeuw, M. Sharir, J. Snoeyink, and B. Speckmann. Efficient Algorithms for Maximum Regression Depth. Discrete Comput. Geom. (to appear).

10. S. Langerman and W. Steiger. Computing a Maximal Depth Point in the Plane. Proc. Japan Conference on Discrete and Computational Geometry, Tokyo, 2000.

11. J. Matoušek. Computing the Center of a Planar Point Set. In Discrete and Computational Geometry: Papers from the DIMACS Special Year (J. E. Goodman, R. Pollack, and W. Steiger, eds.), pp. 221-230. American Mathematical Society, Providence, RI, 1992.

12. P. Rousseeuw and M. Hubert. Depth in an Arangement of Hyperplanes. Discrete Comput. Geom. 22(2) (1999), 167-176.

13. P. Rousseeuw and M. Hubert. Regression Depth. J. Amer. Statist. Assoc. 94 (1999), 388-402.

14. W. Steiger and R. Wenger. Hyperplane Depth and Nested Simplices (extended abstract). Proc. 10th Canadian Conference on Computational Geometry, McGill University, Montreal, 1998.

15. J. Tukey. Mathematics and the Picturing of Data. Proc. International Conference of Mathematicians, Vancouver, 1971.

Received September 7, 2000, and in revised form April 10, 2002. Online publication July 15, 2003.

Note added in proof. The result in [10] is improved to $O\left(n(\log n)^{3}\right)$ in S. Langerman and W. Steiger, Optimization in Arrangements, Lecture Notes in Computer Science 2607, Springer-Verlag, Berlin, pp. 50-61, 2003. 